\title{
Efeito do Gás de Proteção e do Modo de Transferência na Aplicação da Liga 625 em Aço Carbono
}

\author{
(Effect of Shielding Gas and Transfer Mode on the Application of 625 Alloy in Carbon Steel)
}

\author{
Carlos Eduardo Iconomos Baixo', Jair Carlos Dutra ${ }^{l}$ \\ ${ }^{1}$ Universidade Federal de Santa Catarina, Dpto. Eng. Mecânica/ LABSOLDA, Florianópolis, Brasil. \\ carlosbaixo@matrix.com.br, jdutra@labsolda.ufsc.br
}

\begin{abstract}
Resumo
Neste trabalho foram analisados os efeitos do gás de proteção (Ar, Ar/CO, Ar/O2 e Ar/He/O ${ }_{2}$ ) e do modo de transferência (curtocircuito e corrente pulsada) utilizando o processo MIG/ MAG com arame ER-NiCrMo-3 para produzir depósitos sobre chapa assim como o passe de raiz em juntas em V produzidas em aço ABNT 1020. Os resultados mais favoráveis na soldagem do passe de raiz foram obtidos na soldagem com corrente pulsada, empregando uma junta em V com abertura de $90^{\circ}$ e atmosfera de Ar $+25 \%$ CO . A utilização de atmosferas de proteção com $20 \%$ Hélio e baixo percentual de gás ativo não produziu os resultados esperados, sendo eficaz somente na soldagem com deposição sobre chapa, porém tímida em produzir resultados satisfatórios na solda do passe de raiz. Resultados ruins foram obtidos na soldagem com transferência por curto-circuito, independente do gás de proteção, e quando utilizada atmosfera composta por Argônio puro, independente do modo de transferência ajustado.
\end{abstract}

Palavras-Chave: Ligas de Níquel, Ligas 625, ER NiCrMo-3, MIG/ MAG, Soldagem de Raiz, Revestimento.

\begin{abstract}
In this study the effect of shielding gas ( $\mathrm{Ar}, \mathrm{Ar} / \mathrm{CO}_{2}, \mathrm{Ar} / \mathrm{O}_{2}$ and Ar/He/O${ }_{2}$ ) and transfer mode (short-circuit and pulsed current) in MIG/MAG welding with ER-NiCrMo-3 wire to produce bead-on-plate deposits and root passes in "V" joints of ABNT 1020 steel were analyzed. The most favorable results in root pass welding were obtained with pulsed current, employing a " $V$ " joint with a $90^{\circ}$ aperture and $\mathrm{Ar}+25 \% \mathrm{CO}_{2}$ atmosphere. The use of shielding atmospheres with $20 \%$ helium and a low percentage of active gas did not produce the expected results, being effective only in bead-on-plate weld deposition. However, it was not very effective to produce satisfactory results in root pass welding. Poor results were obtained in the welding with short-circuit transfer regardless of the used shielding gas, and when an atmosphere of pure argon was used, regardless of the mode of transfer.
\end{abstract}

Key-Words: Nickel Alloys, 625 Alloys, ER NiCrMo-3, GMAW, Root Welding, Recovery.

\section{Introdução}

Nas últimas décadas tem crescido a utilização de aço revestido para aumentar à resistência a corrosão mantendo os custos de fabricação em níveis inferiores aos requeridos pela utilização de material mais nobre, como aço inoxidável. Para tanto, várias técnicas tem sido empregadas, utilizando desde revestimentos poliméricos até revestimentos metálicos, estes produzidos por processos como laminação dupla, revestimento com chapa ou depositados por processos como aspersão térmica ou soldagem a arco. Além dos aços inoxidáveis, tem sido fortemente considerada a utilização de ligas de Níquel, em especial da liga 625, devido sua elevada resistência à corrosão-fadiga.

Assim como outros revestimentos aplicados por processos de soldagem a arco, caso dos revestimentos resistentes à cavitação, a obtenção de sucesso na aplicação da liga 625 envolve a escolha

(Recebido em 20/11/2008; Texto Final em 05/09/2009). de processos e técnicas de deposição que permitam conciliar taxa de deposição com a produção de soldas com baixa diluição e livres de defeitos. $\mathrm{O}$ elevado custo envolvido nestas operações, em especial devido ao valor comercial do revestimento, tem levado à opção por processos com capacidade de produzir soldas de elevada qualidade, ficando em um plano secundário um aspecto como a taxa de deposição. Por enquadrarem de forma plena nestes requisitos, surgem como escolha freqüente para a aplicação da liga 625 processos como o TIG com alimentação de arame [1] e o Plasma com adição de metal, tanto forma de arame [2], quanto na forma particulada [3,4].

Diante da crescente expansão da demanda por chapas e equipamentos revestidos com ligas 625, surge espaço para a identificação de novos processos e procedimentos de soldagem que permitam ampliar as opções já existentes, adicionando novas técnicas que permitam aumentar o nível de produção ou habilitar processos de fácil utilização em ambiente industrial. Inserido neste contexto, o processo $\mathrm{MIG} / \mathrm{MAG}$, em particular o modo com corrente pulsada, apresenta vantagens comprovadas na aplicação de revestimentos especiais resistentes a cavitação, como a simplicidade de operação, capacidade de produção de 
soldas livres de defeitos e com baixa diluição [5]. Apesar do seu potencial, tem sido tímida a utilização do processo na aplicação de revestimentos com ligas 625, pois, com raras exceções [1,6], o processo tem sido apresentado como aplicável quase que exclusivamente por catálogos de fabricantes de ligas resistentes à corrosão ou de gás de proteção.

Este trabalho se insere em uma pesquisa desenvolvida com o objetivo de formular procedimentos de soldagem para a aplicação da liga 625 no revestimento da superfície interna de tubulações de petróleo, para posterior usinagem do bizel e soldagem dos passes de raiz e quente da junta, também utilizando o arame AWS SFA5.14 ER NiCrMo-3, consumível adequado à soldagem das ligas 625 pelo processo MIG/MAG. Nos primeiros ensaios, a utilização dos processos Eletrodo Revestido e TIG não introduziram maior dificuldade a obtenção de bons resultados. Entretanto, os primeiros ensaios realizados com o processo MIG/MAG com transferência por curto-circuito em atmosfera rica em Argônio $\left(\mathrm{CO}_{2}<5 \%\right)$ demonstraram que a baixa molhabilidade seria um problema a ser enfrentado. Diante da necessidade de aumentar a molhabilidade, o modo de transferência e a composição do gás de proteção se tornam importantes elementos de análise. No tocante ao modo de transferência, o objetivo principal foi o de verificar a possibilidade de utilizar a corrente pulsada em substituição ao curto-circuito, dada à tendência desta última a apresentar menor aporte de calor quando comparada a outros modos de transferência no mesmo valor de corrente média. Quanto à atmosfera de proteção, a pesquisa procurou identificar o efeito produzido pela utilização de misturas ternárias $(\mathrm{Ar} / \mathrm{He} /$ $\mathrm{O}_{2}$ ), frequentemente recomendado para aplicações com ligas 625 , assim como misturas binárias $\left(\mathrm{Ar} / \mathrm{O}_{2} \mathrm{e} \mathrm{Ar} / \mathrm{CO}_{2}\right)$, estendendo a pesquisa à aplicação de gases ativos em teores até superiores àqueles frequentemente utilizados na soldagem com do Inconel 625 .

\section{Materiais e Métodos}

Para a realização dos ensaios, foi utilizada fonte de soldagem MIG/ MAG com capacidade de operar nos modos de transferência curto-circuito, a corrente pulsada, como alternativa à soldagem com transferência por curto-circuito, e, em menor escala, a transferência spray. No tocante aos gases, foi utilizada proteção composta exclusivamente por gás inerte (Argônio puro), por misturas binárias $\left(\mathrm{Ar} / \mathrm{CO}_{2}\right.$ e $\left.\mathrm{Ar} / \mathrm{O}_{2}\right)$ e por misturas ternárias $\left(\mathrm{Ar} / \mathrm{He} / \mathrm{O}_{2}\right)$. As misturas binárias a base de Argônio foram escolhidas pelo seu efeito sobre a molhabilidade dos depositados. A presença de Oxigênio na mistura, seja na forma pura $\left(\mathrm{O}_{2}\right)$ ou combinada $\left(\mathrm{CO}_{2}\right)$, pode reduzir a tensão superficial do metal fundido, conferindo maior molhabilidade ao banho metálico.

Uma proposta diferente para a composição da atmosfera de proteção vem das misturas ternárias, onde Oxigênio é adicionado em percentuais da ordem de 1 a 3\%, para minimizar os efeitos de oxidação, e a presença de He é aumentada para percentuais iguais ou superiores a $20 \%$, em detrimento ao gás Ar, para modificar a geometria da poça de fusão. Neste sentido, Glickstein [7] identificou que a presença de Hélio na mistura aumenta a intensidade do campo elétrico e eleva a condutividade térmica da descarga, o que resulta em arcos mais largos e distribuições de temperatura mais elevadas em relação às descargas produzidas em Argônio puro.

Para produzir as misturas binárias utilizadas nos ensaios foram empregados misturadores de gás Witt para $\mathrm{Ar} / \mathrm{CO}_{2}$ e $\mathrm{Ar} / \mathrm{O}_{2}$, enquanto para a mistura ternária foi utilizada mistura comercial $\left(79 \% \mathrm{Ar}+20 \% \mathrm{He}+1 \% \mathrm{O}_{2}\right)$. Na tabela 1 é apresentado o domínio dos experimentos realizados, envolvendo a composição do gás, os modos de transferência e o tipo de posição (deposito sobre chapa e deposito em junta).

Tabela 1. Tabela com domínio dos experimentos realizados.

\begin{tabular}{|l|c|c|c|c|c|}
\hline Gás/ Modo Transferência & $\mathrm{CC}(1)$ & Spray & Pulsado & DSC (2) & DJ (2) \\
\hline $100 \% \mathrm{Ar}$ & $\mathrm{X}$ & $\mathrm{X}$ & $\mathrm{X}$ & $\mathrm{X}$ & \\
\hline $\mathrm{Ar}+(5,15$ e 25$) \% \mathrm{CO} 2$ & $\mathrm{X}$ & & $\mathrm{X}$ & $\mathrm{X}$ & $\mathrm{X}$ \\
\hline $\mathrm{Ar}+(5,15$ e 25$) \% \mathrm{O} 2$ & $\mathrm{X}$ & & $\mathrm{X}$ & $\mathrm{X}$ & $\mathrm{X}$ \\
\hline $\mathrm{Ar}+20 \% \mathrm{He}+1 \% \mathrm{O} 2$ & $\mathrm{X}$ & & $\mathrm{X}$ & $\mathrm{X}$ & $\mathrm{X}$ \\
\hline
\end{tabular}

Notas: 1 - CC: Curto-Circuito; 2 - DSC: Deposito Sobre Chapa; DJ: Deposito em Junta.

Os ensaios envolveram deposição sobre chapa de aço e a soldagem do passe de raiz em chanfro sem o uso de cobrejunta, conduzidos na posição plana, com condução da pistola de soldagem sendo feita por um soldador treinado. Os depósitos sobre chapa foram conduzidos corpos de prova com dimensões de 150 x $200 \mathrm{~mm}(\mathrm{CxL})$, espessuras de 8,0 e $10,0 \mathrm{~mm}$, em chapas de aço ABNT 1020. Para as soldas de raiz, foram montadas corpos de prova com junta em "V" a partir de chapas de aço ABNT 1020 com dimensões de 150 x $100 \mathrm{~mm}$ (CxL), espessuras de 8,0 e $10,0 \mathrm{~mm}$, ângulos de bizel de $30^{\circ}$ e de $45^{\circ}$, face da raiz (frequentemente denominada como nariz) de $1,0 \mathrm{~mm}$ e diferentes valores de folga (1,0 a 2,0 mm). Todos os ensaios foram realizados utilizando o arame AWS SFA-5.14 ER
NiCrMo-3 de 1,2 mm de diâmetro (Tabelas 2 e 3).

Com exceção ao modo de transferência por spray, os ensaios foram realizados com ajuste de parâmetros para produzir uma corrente média de $100 \mathrm{~A}$, valor este de corrente suficiente para realizar a soldagem do passe de raiz em todas as posições de soldagem. Embora existindo variações de cordão para cordão em virtude da condução manual da soldagem, a velocidade de soldagem foi mantida em valores da ordem de $3,3 \mathrm{~mm} / \mathrm{s}$. Independente de ser deposito sobre chapa ou em junta, as soldas foram conduzidas no sentido de empurrar o material, com ângulo de arraste de aproximadamente $30^{\circ}$ e ângulo de incidência de $90^{\circ}$ em relação à superfície da chapa. 
Tabela 2. Composição química da liga 625 [8].

\begin{tabular}{|c|c|c|c|c|c|c|c|c|c|c|c|c|}
\hline $\begin{array}{c}\mathrm{Ni} \\
(\%)\end{array}$ & $\begin{array}{c}\mathrm{Cr} \\
(\%)\end{array}$ & $\begin{array}{c}\mathrm{Mo} \\
(\%)\end{array}$ & $\begin{array}{c}\mathrm{Nb} \\
(\%)\end{array}$ & $\begin{array}{c}\mathrm{C} \\
(\%)\end{array}$ & $\begin{array}{c}\mathrm{Fe} \\
(\leq \%)\end{array}$ & $\begin{array}{c}\mathrm{Si} \\
(\leq \%)\end{array}$ & $\begin{array}{c}\mathrm{Al} \\
(\leq \%)\end{array}$ & $\begin{array}{c}\mathrm{Ti} \\
(\leq \%)\end{array}$ & $\begin{array}{c}\mathrm{Mn} \\
(\leq \%)\end{array}$ & $\begin{array}{c}\mathrm{Cu} \\
(\leq \%)\end{array}$ & $\begin{array}{c}\mathrm{S} \\
(\leq \%)\end{array}$ & $\begin{array}{c}\mathrm{P} \\
(\leq \%)\end{array}$ \\
\hline Bal. & $\begin{array}{c}20,0 \\
\mathrm{a}\end{array}$ & $\begin{array}{c}8,0 \\
\mathrm{a}\end{array}$ & $\begin{array}{c}3,15 \\
\mathrm{a}\end{array}$ & $\begin{array}{c}0,03 \\
\mathrm{a}\end{array}$ & 5,0 & 0,5 & 0,4 & 0,4 & 0,5 & 0,5 & 0,015 & 0,015 \\
\hline
\end{tabular}

Tabela 3. Valores para a composição química do Arame ER NiCrMo-3 [9].

\begin{tabular}{|c|c|c|c|c|c|c|c|c|c|c|c|c|}
\hline $\begin{array}{c}\mathrm{Ni} \\
(\geq \%)\end{array}$ & $\begin{array}{c}\mathrm{Cr} \\
(\%)\end{array}$ & $\begin{array}{c}\mathrm{Mo} \\
(\%)\end{array}$ & $\begin{array}{c}\mathrm{Nb}+\mathrm{Ta} \\
(\%)\end{array}$ & $\begin{array}{c}\mathrm{C} \\
(\leq \%)\end{array}$ & $\begin{array}{c}\mathrm{Fe} \\
(\leq \%)\end{array}$ & $\begin{array}{c}\mathrm{Si} \\
(\leq \%)\end{array}$ & $\begin{array}{c}\mathrm{Al} \\
(\leq \%)\end{array}$ & $\begin{array}{c}\mathrm{Ti} \\
(\leq \%)\end{array}$ & $\begin{array}{c}\mathrm{Mn} \\
(\leq \%)\end{array}$ & $\begin{array}{c}\mathrm{Cu} \\
(\leq \%)\end{array}$ & $\begin{array}{c}\mathrm{S} \\
(\leq \%)\end{array}$ & $\begin{array}{c}\mathrm{P} \\
(\leq \%)\end{array}$ \\
\hline 58,0 & $\begin{array}{c}20,0 \\
\mathrm{a}\end{array}$ & $\begin{array}{c}8,0 \\
\mathrm{a}\end{array}$ & $\begin{array}{c}3,15 \\
\mathrm{a}\end{array}$ & 0,10 & 5,0 & 0,50 & 0,40 & 0,40 & 0,50 & 0,50 & 0,015 & 0,020 \\
\hline
\end{tabular}

\section{Resultados e Discussões}

\subsection{Depósitos sobre Chapa}

Com exceção a soldagem com transferência por spray, à utilização de Argônio puro na soldagem MIG/MAG com arame ER-NiCrMo-3 se mostrou impraticável. No modo curtocircuito, apesar de uma transferência estável e de uma baixa incidência de respingos, a utilização de Argônio puro resultou em depósitos sem molhabilidade, independente da corrente ajustada. Tentativas de reduzir a velocidade de soldagem para valores inferiores a $3,3 \mathrm{~mm} / \mathrm{s}$, para aumentar as dimensões da poça de fusão, ou a aplicação de técnicas de tecimento, para aumentar a largura dos depósitos, não produziram efeito sobre o ângulo de molhabilidade. O mesmo problema foi observado na soldagem com corrente pulsada. Nesta situação, mesmo utilizando parâmetros de pulso totalmente distintos como 300A/ $4,0 \mathrm{~ms}$ ou $400 \mathrm{~A} / 2,8 \mathrm{~ms}$, os depósitos produzidos em atmosfera de Argônio puro apresentaram baixa molhabilidade (Figura 1). Assim como na transferência por curto-circuito, a utilização de técnicas de tecimento resultou no aparecimento de cordões com maior largura, porém, sem alteração no ângulo de molhabilidade na borda dos depósitos. Resultados mais favoráveis foram obtidos na soldagem com transferência por spray, onde, devido ao elevado valor da corrente, os cordões apresentaram ângulo de molhabilidade aceitável. Entretanto, os níveis de corrente aplicados, na faixa de $200 \mathrm{~A}$ limitam sua utilização à soldagem na posição plana e peças espessas, caso se comprove que a elevada energia não resulta na produção de depósitos com elevada diluição.

Nos ensaios com misturas binárias, verificou-se que adições de 5 e de $15 \%$ de $\mathrm{CO}_{2}$ ao Argônio, apesar de não produziram alteração na estabilidade do destacamento das gotas, também não modificaram a geometria da solda, que manteve o perfil de baixa molhabilidade (Figura 2). Resultados favoráveis foram obtidos com adições de $25 \%$ de $\mathrm{CO}_{2}$, em especial na soldagem com corrente pulsada, visto a melhora produzida na geometria da solda (Figura 3), sem produzir, contudo, alterações na estabilidade da transferência metálica. Já na soldagem com transferência por curto-circuito, os resultados não foram favoráveis visto a tendência de baixa molhabilidade dos depósitos.

Ensaios conduzidos com atmosfera binária de $\mathrm{Ar}+\mathrm{O}_{2}$ demonstraram que a adição de $25 \%$ de Oxigênio ao Argônio torna a mistura eficaz no aumento da molhabilidade dos depósitos, mas apresenta como desvantagem, a tendência de produzir uma transferência metálica mais instável. Em especial para a soldagem em corrente pulsada, a adição de $25 \%$ de Oxigênio produziu uma transferência caracterizada pelo surgimento de mais de uma gota por pulso e/ ou pelo crescimento excessivo do glóbulo antes do destacamento. Em ambos as situações, gotas metálicas acabaram por ser ejetadas para fora do ambiente do arco, caracterizando a formação de salpicos. Tentativas de alterar os parâmetros de pulsação da corrente foram incapazes de eliminar a ocorrência destes glóbulos indesejáveis. Além dos problemas de relacionados com a instabilidade da transferência, os depósitos obtidos sob proteção gasosa de $\mathrm{Ar}+\mathrm{O}_{2}$ apresentaram uma coloração mais escura, além de produzir depósitos com aspecto escorrido. De forma semelhante à mistura $\mathrm{Ar}+\mathrm{CO}_{2}$, a adição de percentuais de Oxigênio de 5 e $15 \%$ ao Argônio não produziram mudanças significativas na geometria da solda, independente do modo de transferência empregado para a soldagem.

Nos depósitos sobre chapa com transferência por curtocircuito protegida pela mistura ternária (Figura 4), apesar da presença de Hélio e Oxigênio na composição, não houve uma alteração significativa nos resultados em relação às soldas produzidas com atmosfera de Argônio puro ou com adições de $\mathrm{CO}_{2}$ e $\mathrm{O}_{2}$. Resultados mais favoráveis foram obtidos na transferência com corrente pulsada, onde a utilização da mistura ternária $\mathrm{Ar} / \mathrm{He} / \mathrm{O}_{2}$ produziu depósitos com molhabilidade muito superior àquela resultante da soldagem com transferência por curto-circuito, apesar do valor da corrente média, em ambos os casos, ser da mesma magnitude (Figura 5). 


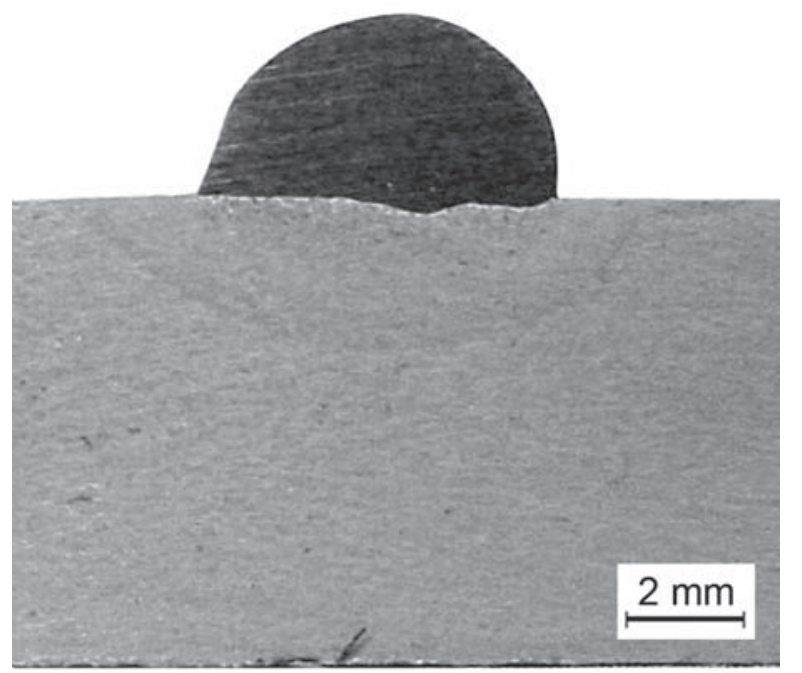

Figura 1. Macrografia depósito sobre chapa produzido pelo processo MIG com corrente pulsada. Dados: Ip: $300 \mathrm{~A}$; tp: 4,0 ms; Ib: 45; tb: 14,6 ms; Va: 0,052 m/ s; Im: 104 A; Um: 20,4 V; Vs: 3,0 mm/s; Aporte: 0,7 kJ/ mm; Gás: 100\%Ar.

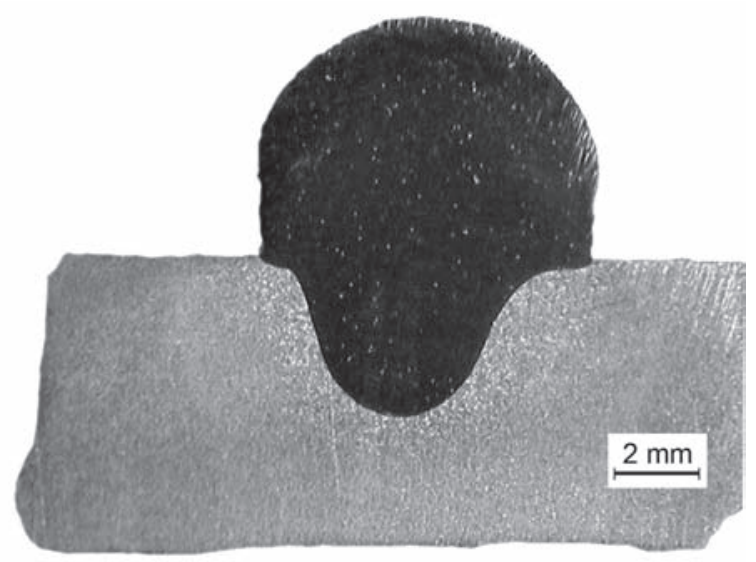

Figura 2. Macrografia depósito sobre chapa produzido pelo processo MAG com corrente pulsada. Dados: Ip: $300 \mathrm{~A}$; tp: 4,0 ms; Ib: 45; tb: 14,6 ms; Va: 0,052 m/ s; Im: 102 A; Um: 20,3 V; Vs: 3,0 mm/s; Aporte: 0,7 kJ/ mm; Gás: Ar+15\% $\mathrm{CO}_{2}$.

Considerando que a soldagem com transferência por spray produziu boa molhabilidade mesmo quando utilizado gás Argônio puro, o aporte de energia se mostra importante na obtenção de molhabilidade nos depósitos sobre chapa. Para os depósitos produzidos com transferência por curto-circuito em baixo aporte $(0,5 \mathrm{~kJ} / \mathrm{mm})$, tanto as misturas binária $\left(\mathrm{Ar} / \mathrm{O}_{2}\right.$ e $\left.\mathrm{Ar} / \mathrm{CO}_{2}\right)$ quanto ternárias $\left(\mathrm{Ar} / \mathrm{He} / \mathrm{O}_{2}\right)$ se mostraram ineficazes em alterar a molhabilidade. Já na soldagem com corrente pulsada, onde o aporte de calor médio foi da ordem de $0,8 \mathrm{~kJ} / \mathrm{mm}$, independente da mistura ser binária ou ternária, houve a formação de depósitos com geometria favorável. Seguindo a tendência, os melhores resultados obtidos correspondem aos depósitos sobre chapa com um aporte de calor de aproximadamente 1,3 kJ/ mm (202 A/; $29,5 \mathrm{~V} / 4,5 \mathrm{~mm} / \mathrm{s}$ ), obtidos na soldagem com transferência por spray mesmo com Argônio puro.

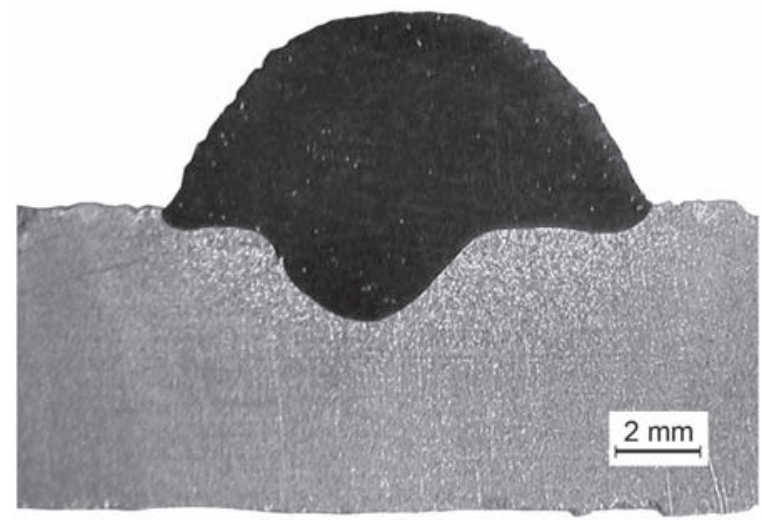

Figura 3. Macrografia depósito sobre chapa produzido pelo processo MAG com corrente pulsada. Dados: Ip: $300 \mathrm{~A}$; tp: 4,0 ms; Ib: 45; tb: 14,6 ms; Va: 0,055 m/ s; Im: $102 \mathrm{~A}$; Um: 23,3 V; Vs: 3,0 mm/s; Aporte: 0,8 kJ/ mm; Gás: Ar+25\% $\mathrm{CO}_{2}$.

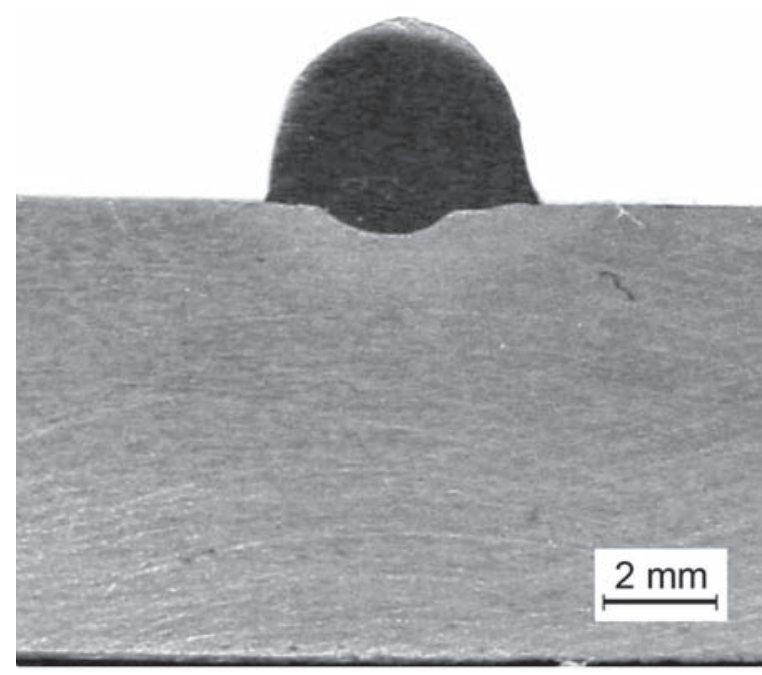

Figura 4. Macrografia de deposito sobre chapa com transferência por curto-circuito e atmosfera ternária. Dados: Va = 3,6 m/ min; Im = $102 \mathrm{~A} ; \mathrm{Um}=17 \mathrm{~V} ; \mathrm{Vs}: 3,0 \mathrm{~mm} / \mathrm{s}$; Aporte: $0,58 \mathrm{~kJ} / \mathrm{mm}$; Gás: $\mathrm{Ar}+20 \% \mathrm{He}+1 \% \mathrm{O}_{2}$.

\subsection{Depósitos em junta (Passe de Raiz)}

Com relação à soldagem do passes de raiz em juntas de 60 e $90^{\circ}$, a transferência metálica por curto-circuito produziu uma transferência estável, porém de difícil aplicação em função da baixa capacidade de reprodução dos depósitos. Durante os ensaios, combinações de parâmetros que resultaram em geometrias aceitáveis em um primeiro teste produziram, em uma segunda tentativa, depósitos com falta de penetração total ou com má formação geométrica, esta caracterizada pela falta de simetria no depósito (Figura 6). Tentativas de aumentar a folga da junta, reduzir ângulo de abertura ou diminuir o tamanho de nariz não produziram resultados satisfatórios, em especial pela baixa fluidez da poça de fusão, que limitou o aumento na velocidade de deslocamento da pistola de soldagem. Com transferência por curto-circuito, mostrou-se frequente a formação de depósitos 
com excesso de penetração (Figura 7), problema que persistiu independente do gás de proteção utilizado durante a soldagem. A adição de $\mathrm{CO}_{2}$ ou $\mathrm{O}_{2}$ ao Argônio produziu uma melhora na molhabilidade dos depósitos produzidos com transferência por curto-circuito, porém foi incapaz de melhorar a baixa repetitividade do processo. Mesmo a utilização de mistura ternária não produziu resultados satisfatórios, igualmente em função da baixa repetitividade nos depósitos produzidos com transferência por curto-circuito. Computados os resultados obtidos, o baixo índice de acertos retrata de forma fiel a dificuldade em utilizar o processo MIG/MAG com transferência por curto-circuito na soldagem do passe de raiz, não somente pela baixa molhabilidade, mas também pela elevada dificuldade em garantir a repetitividade nas dimensões do reforço da raiz.

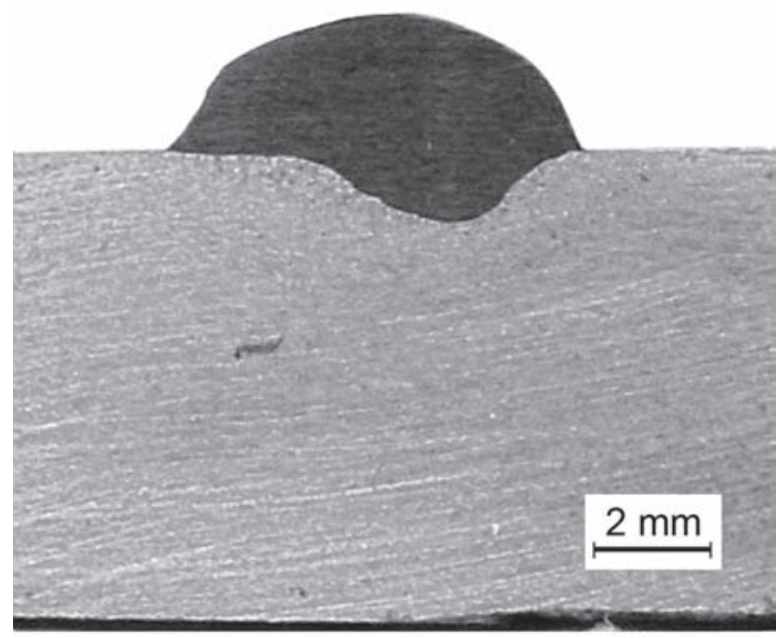

Figura 5. Macrografia de deposito sobre chapa com transferência com corrente pulsada e atmosfera ternária. Dados: Ip: 300 A; tp: 4,0 ms; Ib: 45; tb: 14,6 ms; Va: 0,055 m/ s; Im: 102 A; Um: 23,3 V; Vs: 3,0 mm/ s; Aporte: 0,8 kJ/ mm; Gás:

$$
\mathrm{Ar}+20 \% \mathrm{He}+1 \% \mathrm{O}_{2} \text {. }
$$

Resultados mais favoráveis na soldagem do passe de raiz com o processo MIG/ MAG foram obtidos operando com corrente pulsada, onde depósitos com geometria adequada para soldas com múltiplos passes foram obtidos empregando junta em V com abertura de 90 o e atmosfera de $\mathrm{Ar}+25 \% \mathrm{CO}_{2}$ (fig. 8). Além da geometria mais favorável, foram observados menos problemas relacionados à repetitividade, tanto no que tange ao excesso, quanto a uma falta de penetração. Entretanto, apesar da combinação corrente pulsada/ $\mathrm{Ar}+25 \% \mathrm{CO}_{2}$ ser a que produziu menos problemas relacionados com a geometria do passe de raiz, a obtenção de uma raiz uniformidade impôs alto grau de dificuldade, em especial quando comparados aos processos TIG e Eletrodos Revestidos, estes com maior facilidade para a produção de uma raiz satisfatória. Já a soldagem empregando corrente pulsada e a mistura ternária com $\mathrm{Ar}, \mathrm{He}$ e $\mathrm{O}_{2}$ não produziu bons resultados na soldagem em chanfro, devido à irregularidade dos depósitos produzidos. Tentativas de atuar nos parâmetros de pulsação ou geometria da junta não permitiram resolver o problema e, com isto, obter soldas de qualidade aceitável. Para esta combinação de modo de transferência e gás de proteção, soldas com geometria favorável ficaram limitadas aos ensaios envolvendo deposição sobre chapa.

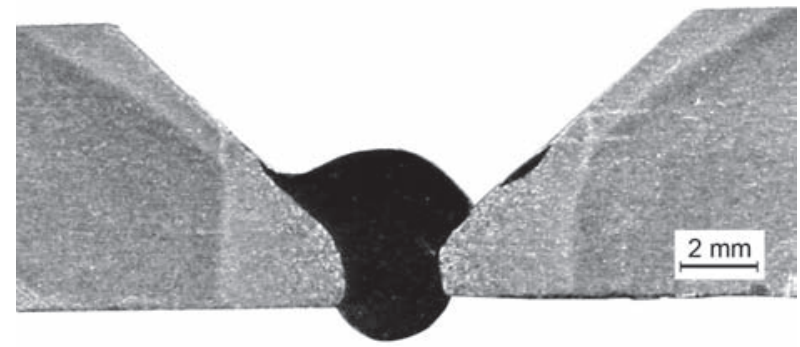

Figura 6. Formação geométrica com falta de simetria do passe de raiz na soldagem MAG com transferência por curto-circuito. Dados: Va = 3,6 m/ min; Im = $102 \mathrm{~A} ; \mathrm{Um}=17 \mathrm{~V}$; Vs: 3,2 mm/ s; Aporte: 0,5 kJ/ mm; Gás: $\mathrm{Ar}+25 \% \mathrm{CO}_{2}$. Chanfro de $90^{\circ}$.

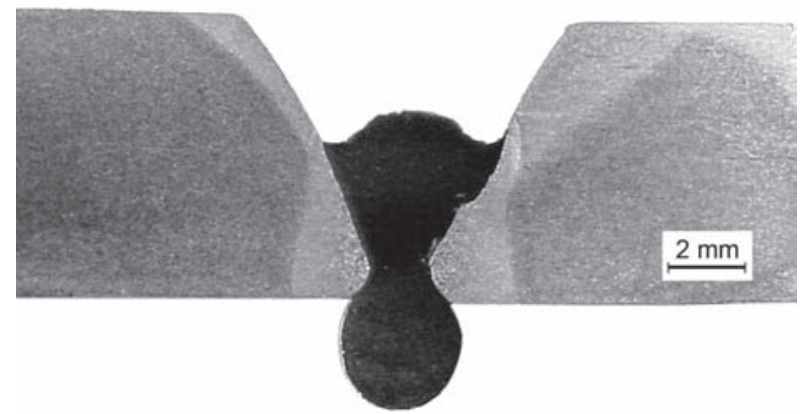

Figura 7. Formação de um reforço excessivo ne solda do passe de raiz na soldagem MAG com transferência por curto-circuito. Dados: $\mathrm{Va}=3,6 \mathrm{~m} / \mathrm{min} ; \mathrm{Im}=102 \mathrm{~A} ; \mathrm{Um}=17 \mathrm{~V}$; Vs: 3,2 mm/ s; Aporte: 0,5 kJ/ mm; Gás: Ar+25\% $\mathrm{CO}_{2}$. Chanfro de $60^{\circ}$.

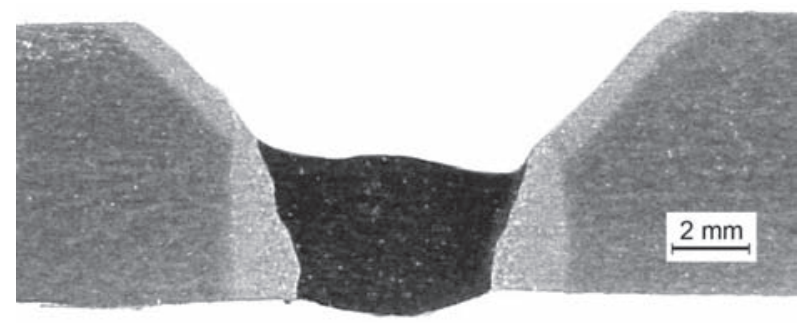

Figura 8. Macrografia do passe de raiz na soldagem MAG com transferência com corrente pulsada. Dados: Ip: $300 \mathrm{~A}$; tp: 4,0 ms; Ib: 45; tb: 14,6 ms; Va: 0,055 m/ s; Im: 102 A; Um: 23,3 V; Vs: $3,2 \mathrm{~mm} / \mathrm{s}$; Aporte: 0,8 kJ/ mm; Gás: $\mathrm{Ar}+25 \% \mathrm{CO}_{2}$.

No que tange a utilização da soldagem MIG/MAG para a aplicação de revestimento, apesar dos bons resultados obtidos com combinação $\mathrm{MAG}$ pulsado/ $\mathrm{Ar}+25 \% \mathrm{CO}_{2}$ nos depósitos sobre chapa, a literatura é enfática na limitação do percentual de gás ativo passível de ser utilizado na soldagem de ligas de Níquel. Para o NDI [10], a soldagem MIG/ MAG de ligas de Níquel deve ser executada em atmosferas onde a presença de gases inertes (Ar ou Ar/He) seja igual ou superior a 97,5\% para evitar o aparecimento de depósitos superficiais de alta dureza, formados a partir de reações de oxidação do $\mathrm{Al}$ e Ti, cuja presença em níveis relativamente baixos busca minimizar o efeito de endurecimento por envelhecimento [11]. Entretanto, por serem compostos refratários e de alto poder de adesão, a presença de 
óxidos de $\mathrm{Al}$ e Ti exige remoção mecânica da superfície do metal depositado, caso a soldagem envolva múltiplos passes. A Avesta Welding [12] igualmente recomenda a adição de 1 a $3 \%$ de $\mathrm{CO}_{2}$ à mistura $\mathrm{Ar}+30 \%$ He para estabilizar o arco durante a soldagem, sugerindo que percentuais maiores de $\mathrm{CO}_{2}$ podem provocar a oxidação da superfície e a inclusão de Carbono no metal através da poça fundida. Na mesma linha de recomendação, porém sem apresentar justificativa, a Kobelco [13] e a Praxair [14] sugerem atmosferas formadas pelos gases $\mathrm{Ar}, \mathrm{Ar} / \mathrm{He}$ ou $\mathrm{Ar} / \mathrm{He} /$ $\mathrm{CO}_{2}$, neste último sempre limitando o percentual de gás ativo na mistura a valores da ordem de 1 a $3 \%$. Situação bastante diversa é encontrada na utilização de metal de adição para as ligas 625 na forma de arame tubular, onde a presença de $25 \%$ de $\mathrm{CO} 2$ na atmosfera gasosa é considerada fundamental para a obtenção de depósitos aceitáveis $[10,13,15]$. Confrontando as justificativas apresentadas para a limitação do percentual de $\mathrm{CO}_{2}$ para a soldagem com o arame ER NiCrMo-3, é possível verificar que os fabricantes de arame tubular para as ligas 625 não indicam a presença de $\mathrm{Ti}$ ou $\mathrm{Al}$ (Tabela 4) na composição do metal depositado [15,16], o que contribui para a explicação acerca do efeito deletério da presença óxidos refratários originados pela adição de $\mathrm{CO}_{2}$ ao gás de proteção. Entretanto, é necessário salientar a existência de fabricantes de arame tubular para ligas 625 que sugerem a realização da soldagem com atmosfera contendo $25 \%$ de $\mathrm{CO}_{2}$ apesar da existência, ainda que em percentual baixo, de Ti e Al na composição do metal depositado [Tabela 5].

Tabela 4. Valores para a composição química ${ }^{(1)}$ do arame tubular compatível para a soldagem da liga 625.

\begin{tabular}{|c|c|c|c|c|c|c|c|c|c|c|c|c|}
\hline Ref. & $\begin{array}{c}\mathrm{Cr} \\
(\%)\end{array}$ & $\begin{array}{c}\mathrm{Mo} \\
(\%)\end{array}$ & $\begin{array}{c}\mathrm{Nb} \\
(\%)\end{array}$ & $\begin{array}{c}\mathrm{C} \\
(\%)\end{array}$ & $\begin{array}{c}\mathrm{Fé} \\
(\leq \%)\end{array}$ & $\begin{array}{c}\mathrm{Si} \\
(\leq \%)\end{array}$ & $\begin{array}{c}\mathrm{Al} \\
(\leq \%)\end{array}$ & $\begin{array}{c}\mathrm{Ti} \\
(\leq \%)\end{array}$ & $\begin{array}{c}\mathrm{Mn} \\
(\leq \%)\end{array}$ & $\begin{array}{c}\mathrm{Cu} \\
(\leq \%)\end{array}$ & $\begin{array}{c}\mathrm{S} \\
(\leq \%)\end{array}$ & $\begin{array}{c}\mathrm{P} \\
(\leq \%)\end{array}$ \\
\hline 13 & 22,0 & 9,2 & 3,8 & 0,03 & 3,0 & 0,3 & $\mathrm{NE}$ & $\mathrm{NE}$ & 0,4 & $\mathrm{NE}$ & $\mathrm{NE}$ & $\mathrm{NE}$ \\
\hline 11 & 21,43 & 9,21 & 3,42 & 0,028 & 4,84 & 0,31 & $\mathrm{NE}$ & $\mathrm{NE}$ & 0,88 & 0,01 & 0,004 & 0,004 \\
\hline 14 & 21 & 9,0 & 3,4 & 0,02 & 0,40 & 0,3 & $\mathrm{NE}$ & $\mathrm{NE}$ & 0,4 & $\mathrm{NE}$ & $\mathrm{NE}$ & $\mathrm{NE}$ \\
\hline
\end{tabular}

(1) - Teor de Níquel: Balanço.

Tabela 5. Valores para a composição química do Arame ER NiCrMo-3 [17].

\begin{tabular}{|c|c|c|c|c|c|c|c|c|c|c|c|c|}
\hline $\begin{array}{c}\mathrm{Ni} \\
(\geq \%)\end{array}$ & $\begin{array}{c}\mathrm{Cr} \\
(\%)\end{array}$ & $\begin{array}{c}\mathrm{Mo} \\
(\%)\end{array}$ & $\begin{array}{c}\mathrm{Nb}+\mathrm{Ta} \\
(\%)\end{array}$ & $\begin{array}{c}\mathrm{C} \\
(\leq \%)\end{array}$ & $\begin{array}{c}\mathrm{Fe} \\
(\leq \%)\end{array}$ & $\begin{array}{c}\mathrm{Si} \\
(\leq \%)\end{array}$ & $\begin{array}{c}\mathrm{Al} \\
(\leq \%)\end{array}$ & $\begin{array}{c}\mathrm{Ti} \\
(\leq \%)\end{array}$ & $\begin{array}{c}\mathrm{Mn} \\
(\leq \%)\end{array}$ & $\begin{array}{c}\mathrm{Cu} \\
(\leq \%)\end{array}$ & $\begin{array}{c}\mathrm{S} \\
(\leq \%)\end{array}$ & $\begin{array}{c}\mathrm{P} \\
(\leq \%)\end{array}$ \\
\hline 64 & 20 & 9 & 3,4 & 0,04 & 0,50 & 0,30 & 0,05 & 0,10 & 0,30 & 0,05 & 0,015 & 0,020 \\
\hline
\end{tabular}

Por outro lado, se a utilização de $\mathrm{Ar} / \mathrm{CO}_{2}$ pode ser vetada para soldas com múltiplos passes devido ao aparecimento de microestruturas duras, eventualmente a mistura poderia ser utilizada para soldas em passe único, uma vez que, nesta situação, a presença dos óxidos na superfície não resultaria em maiores conseqüências aos depósitos. Importante ressaltar que o aparecimento de regiões de microestruturas duras na soldagem das ligas 625 pode ocorrer independentemente do gás de proteção, do modo de transferência ou mesmo do processo de soldagem empregado. Ao investigar a tenacidade à fratura da zona afetada pelo calor/ zona de ligação (ZAC-ZL), de juntas de aço X60 soldado com eletrodo ER NiCrMo-3, Pope e outros [18] demonstraram que essas juntas são susceptíveis a apresentar zonas frágeis localizadas (ZFL), caracterizadas pela presença de microestrutura martensítica com dureza de, aproximadamente, $360 \mathrm{HV}$.

De uma maneira geral, a utilização de atmosferas de proteção com baixo percentual de gás ativo não produziu os resultados esperados na soldagem em junta. Apesar literatura indicar que a presença de He é eficaz na obtenção de depósitos com boa molhabilidade, os resultados encontrados sugerem que adições na faixa de $20 \%$ são eficazes somente na deposição sobre chapa, sendo o percentual tímido em produzir resultados satisfatórios na raiz. Eventualmente, percentuais na faixa de $30 \%$, ou mais, melhorem a molhabilidade a ponto de permitir produzir uma solda de raiz satisfatória com o processo MIG/MAG. Entretanto, diante da dificuldade encontrada mesmo quando utilizada mistura $\mathrm{Ar}+25 \% \mathrm{CO}_{2}$, a utilização do processo para a soldagem em junta merece maior investigação.

Diante do potencial de redução de custos, não somente pelo preço do metro cúbico do gás, mas também pela logística de fornecimento, assume importância que se realize uma análise do real efeito gerado pela utilização de $\mathrm{Ar} / \mathrm{CO} 2$ na formação de microestruturas duras e o seu potencial de risco na fragilização da união soldada visando à aplicação do processo para enchimento/ acabamento em juntas ou revestimento de superfícies por sobreposição. A sugestão para estas aplicações advém da dificuldade encontrada neste trabalho para a aplicação do ER NiCrMo-3 na execução do passe de raiz em juntas produzidas em chapas de aço ABNT 1020, independente do gás ou modo de transferência. Importante citar, igualmente, a necessidade de verificar se a presença de gases ativos na atmosfera pode conduzir a uma queda na resistência corrosão.

\section{Conclusões}

Neste trabalho foram analisados os efeitos do gás de proteção e do modo de transferência MIG/ MAG na soldagem com arame ER NiCrMo-3 com 1,2 mm de diâmetro tanto em depósitos sobre chapa quanto em juntas produzidas em aço ABNT 1020.

Com relação aos depósitos sobre chapa, os resultados obtidos indicam que: 
- a utilização de atmosferas de proteção com 20\% Hélio e baixo percentual de gás ativo se mostrou eficaz somente na soldagem com deposição sobre chapa, produzindo depósitos com boas molhabilidade;

- resultados ruins foram obtidos na soldagem com transferência por curto-circuito, independente do gás de proteção;

- a utilização de atmosfera composta por Argônio puro só se mostrou viável para a soldagem com transferência por spray, quando os depósitos apresentam boa molhabilidade. Em outros modos de transferência (curto-circuito e corrente pulsada) a baixa molhabilidade dos cordões de solda inviabilizou a utilização de Argônio puro mesmo para a soldagem por deposição sobre chapa;

Com relação aos depósitos em junta, observou-se que:

- os resultados mais favoráveis na soldagem do passe de raiz foram obtidos na soldagem com corrente pulsada, empregando uma junta em $\mathrm{V}$ com abertura de $90^{\circ}$ e atmosfera de $\mathrm{Ar}+25 \%$ CO2. Entretanto, apesar da mistura gasosa empregada ter promovido melhora no aspecto da solda, ainda se observou uma dificuldade em manter a uniformidade do reforço do verso da raiz ao longo da junta;

- a utilização de atmosferas de proteção com $20 \%$ Hélio e baixo percentual de gás ativo não produziu os resultados esperados, sendo tímido o seu efeito em melhorar a molhabilidade do passe de raiz;

\section{Referências Bibliográficas}

[1] CAPITANESCU, D. Alloy625 Weld Overlays for Offshore and Onshore Projects. International Symposium on Metallurgy and Applications of Superalloys 718, 625 and Various Derivatives. Ed. The Minerals, Metals \& Materials Society, USA, 1991.

[2] DUTRA, J. C., RIBAS, F., OLIVEIRA, M. A., GOHR JR., R., SILVA, R. H. G. Desenvolvimento dos Processos TIG/ Plasma alimentados automaticamente para aplicação manual em reparos de cavitação em turbinas hidráulicas. In: CITENEL, 2005, Florianópolis. CITENEL, 2005. v. I.

[3] WILSON, I. L. W., GOURLEY, R. G., WALKOSAK, R. M. AND BRUCK, G. J. The Effect of Heat Input on Microstructure and Cracking in Alloy 625 Weld Overllays. International Symposium on Metallurgy and Applications of Superalloys 718, 625 and Various Derivatives. Edited by Edward A. Lima. The Minerals, Metals \& Materials Society, 1991.

[4] DUTRA, J. C. Desempenho do Processo de Soldagem Plasma de Arco Transferido Alimentado com Pó (PTAP) sobre a Resistência à Cavitação. In: XXXI CONSOLDA, 2005, São Paulo. XXXI CONSOLDA, 2005.

[5] BARRA, S. R., DUTRA, J. C. ERIBAS, F. A. C. Avaliação dos Efeitos da Variação dos Parâmetros/ Procedimentos de Soldagem e Automação Sobre a Planicidade e Aspecto Superficial dos Revestimentos Empregados em Turbinas Hidráulicas.. In: XXIV Encontro Nacional da Tecnologia da Soldagem, XI Congresso Latino-Americano e V Íbero-Americano de Soldagem, 1998, Fortaleza - Ce.. XXIV ENTS. São Paulo - SP. : Associação Brasileira de Soldagem, 1998. v. CD.

[6] JOAN L. BARNA, J. L. AND RIVERS, K. B. Improving Recovery Boiler Furnace Reliability With Advanced Materials and Application Methods. Canadian Pulp and Paper Association, 1999.

[7] GLICKSTEIN, S.S. Arc Modelling for Welding Analysis. Arc Physisc and Weld Pool Behaviour. Vol. I - Papers. Ed. The Welding Institute, London, 1980, p.1- 16.

[8] Material Datasheet UNS N06625. Ed. M. Woite GmbH. 2001.

[9] ASME 2007, Section II, Part C. Specification for Nickel and Nickel and Nickel-Alloy Bare Welding Electrodes and Rods. SFA-5.14/ SFA-5.14M.

[10] Nickel Development Institute. Guidelines for the Welded Fabrication of Nickel Alloys for Corrosion-Resistant Services. Canada, 1994. 35 p.

[11] EISELSTEIN, H. L., TILLACK, D. J. The Invention and Definition of Alloy 625. International Symposium on Metallurgy and Applications of Superalloys 718, 625 and Various Derivatives. Edited by Edward A. Lmia. The Minerals, Metals \& Materials Society, 1991.

[12] Avesta Welding. The Avesta Welding Manual - Practice and Products for Stainless Steel Welding, 2004. 300 p.

[13] Kobelco Steel Ltd. Kobelco Welding Handbook - Welding Consumables and Process, 2007. 359 p.

[14] Praxair Technology Inc. Shield Gas - Selection Manual, 1998. 63p.

[15] Stood - Thermadyne Industries Inc. Nickel Flux Cored Wires, 2005.

[16] Welding Alloys Group. GAMMA 625 Technical Data Sheet, 2005.

[17] Special Metals Welding Products Company. INCO-CORED 625DH \& 625AP Flux Cored Wires Technical Data Sheet, 2003.

[18] POPE, A. M., CARDOSO, A, CHIAPIM, C. E. L., NASCIMENTO, J. L., TEIXEIRA, J. C. G., CUNHA, L. D., PAES, M. T. P. Tenacidade à Fratura de Juntas Soldadas de Aços Dissimilares. Em: Encontro Técnico de Soldagem, 1º 2004, Rio de Janeiro. Anais I ENSOLD, Rio de Janeiro, RJ, 2004. 17 p. 